\title{
DETERMINAÇÃO DO LIMIAR DE ANAEROBIOSE DE IDOSOS SAUDÁVEIS: COMPARAÇÃO ENTRE DIFERENTES MÉTODOS
}

\author{
Pozzi LG ${ }^{1}$, Melo RC ${ }^{1}$, Quitério RJ ${ }^{1,2}$, Milan LA ${ }^{3}$, Diniz CAR ${ }^{3}$, Dias TCM ${ }^{3}$, Oliveira L ${ }^{1}$, \\ Silva E ${ }^{2}$ e Catai AM ${ }^{1}$ \\ ${ }^{1}$ Núcleo de Pesquisa em Exercício Físico, Departamento de Fisioterapia, Universidade Federal de São Carlos - UFSCar, \\ São Carlos, SP - Brasil \\ ${ }^{2}$ Faculdade de Ciências da Saúde, Universidade Metodista de Piracicaba, Piracicaba, SP - Brasil \\ ${ }^{3}$ Departamento de Estatística, UFSCar, São Carlos, SP - Brasil \\ Correspondência para: Luis Gustavo Pozzi, Núcleo de Pesquisa em Exercício Físico (NUPEF), Dep. de Fisioterapia, \\ UFSCar, Via Washington Luís, km 235, CP 676, CEP 13565-905, São Carlos, SP - Brasil
}

Recebido: 09/02/2006 - Aceito: 01/06/2006

\begin{abstract}
RESUMO
Objetivo: Determinar o limiar de anaerobiose (LA) pelo método ventilatório (visual gráfico), pelos modelos matemáticos, Heteroscedástico e Hinkley, aplicados aos conjuntos de dados de freqüência cardíaca (FC), RMS do sinal mioelétrico (Root Mean Square) e $\mathrm{VCO}_{2}$ e comparar o LA obtido pelos três métodos. Metodologia: Foram estudados 9 idosos ativos (61,4 $\pm 1,8$ anos) durante teste de exercício físico dinâmico contínuo do tipo rampa, em cicloergômetro, com incrementos de potência variando de 10 a 15 Watts/min. Foram coletados os dados de FC batimento a batimento, eletromiografia de superfície do músculo vasto lateral e variáveis ventilatórias respiração a respiração. Após a aplicação dos modelos matemáticos e identificados os pontos de quebra de comportamento, foram registrados neste momento os valores de potência, $\mathrm{VO}_{2}$ e FC, comparados e correlacionados aos obtidos pelo método visual gráfico (padrão ouro). Foi utilizado o teste de Friedman para comparações múltiplas e o teste de correlação de Spearman (nível de significância de 5\%). Resultados: Não foram encontradas diferenças significantes, em relação ao padrão ouro, entre os valores de potência, $\mathrm{VO}_{2}$ e FC, no momento do LA identificado pelos diferentes modelos. Foram encontradas correlações significantes entre os valores de FC identificados pelos modelos matemáticos entre os valores de $\mathrm{VO}_{2}$ quando identificados pela freqüência cardíaca e de potência somente quando identificada pelo modelo de Hinkley aplicado aos dados de RMS do sinal mioelétrico. Conclusões: No grupo estudado, os modelos matemáticos mostraram-se adequados na determinação não-invasiva do LA, sendo que ambos ajustaram-se melhor aos dados de FC, seguido pela VCO $_{2}$ e RMS.
\end{abstract}

Palavras-chave: limiar de anaerobiose, modelos matemáticos, idosos.

\section{ABSTRACT \\ Determination of Anaerobic Threshold in Healthy Elderly People: Comparison Between Different Methods}

Objective: To determine the anaerobic threshold by the graphic visual ventilatory method and the Hinkley and heteroscedastic mathematical models, applied to heart rate, myoelectric root mean square (RMS) signal and $\mathrm{VCO}_{2}$ datasets, and to compare the anaerobic threshold obtained by the three methods. Method: Nine active elderly subjects were studied (aged $61.4 \pm 1.8$ years) during a ramp-load continuous dynamic physical exercise test on a cycle ergometer, with power ranging from 10 to 15 Watts/ min. Beat-to-beat heart rate data, electromyographic data from the surface of the vastus lateralis muscle, and breath-to-breath ventilatory data were collected. After applying mathematical models and identifying the behavioral shift points, these power levels, heart rates and $\mathrm{VO}_{2}$ values were noted and these were compared and correlated with those obtained by the graphic visual model (gold standard). The Friedman test for multiple comparisons and the Spearman correlation test were utilized (significance level: $5 \%$ ). Results: No significant differences were found in relation to the gold standard, between the power levels, $\mathrm{VO}_{2} \mathrm{values}$ and heart rates at the anaerobic threshold identified by the different models. Significant correlations were found between the heart rates identified by the mathematical models, between the $\mathrm{VO}_{2}$ values identified by the heart rates, and between power rates only when identified by the Hinkley model applied to myoelectric RMS signal data. Conclusion: In this study group, the mathematical models were shown to be adequate for non-invasively determining the anaerobic threshold. Both models worked 
best on the heart rate data, followed by $\mathrm{VCO}_{2}$ and RMS.

Key words: anaerobic threshold, mathematical models, elderly people.

\section{INTRODUÇÃO}

O limiar de anaerobiose (LA) tem sido definido como a intensidade de exercício físico na qual a produção de energia pelo metabolismo anaeróbio predomina em relação ao metabolismo aeróbio, refletindo um aumento na concentração de lactato sangüíneo e na razão lactato/piruvato no músculo ou sangue arterial ${ }^{1}$.

Este parâmetro tem sido utilizado na determinação da capacidade física de um indivíduo e prescrição de treinamento físico ${ }^{1}$, podendo variar em função do grau de treinamento físico ${ }^{2}$ e também da idade, onde ocorre uma diminuição progressiva da capacidade física, tanto por processos fisiológicos como por fatores secundários como o sedentarismo ${ }^{3}$.

A possibilidade de se inferir o limiar de anaerobiose por diferentes metodologias como: o método ventilatório ${ }^{1,3,4,5,6}$, pela resposta da freqüência cardíaca $(\mathrm{FC})^{6,7,8,9,10}$ ou pela eletromiografia de superfície $(\mathrm{EMGs})^{6,11,12,13}$, permitiu a obtenção de resultados satisfatórios quanto à avaliação da capacidade funcional e prescrição de atividade física.

A aplicação de modelos matemáticos para o estudo das respostas de variáveis cardiorrespiratórias e musculares tem sido amplamente utilizada. Assim, Lucía et al. ${ }^{11}$, verificaram que a aplicação de um modelo matemático de regressão linear multisegmentar, aplicado aos valores de RMS do sinal mioelétrico de ciclistas de elite obtidos em teste de esforço do tipo rampa, foi um método eficaz na identificação do limiar de anaerobiose, bem como do ponto de compensação respiratória.

Crescêncio ${ }^{5}$, ao avaliar o comportamento das variáveis ventilatórias de indivíduos adultos sadios, utilizando os modelos visual gráfico, automático (realizado pelo próprio sistema de análise de medidas respiratórias e metabólicas), bissegmentados linear-linear e linear-quadrático para identificar o LA, mostrou a aplicabilidade destes modelos matemáticos.

Trabalhos prévios realizados em nosso laboratório utilizando o modelo matemático de Hinkley aplicado aos dados de $\mathrm{VCO}_{2}$, FC e RMS do sinal mioelétrico em jovens ativos ${ }^{13}$ e indivíduos de meia-idade sedentários ${ }^{6}$ e hipertensos ${ }^{14}$ com objetivo de se identificar o LA e correlacioná-lo com o modelo padrão ouro (visual), mostraram que o modelo apresentouse sensível na detecção deste parâmetro.

No entanto, a avaliação do limiar de anaerobiose por modelos matemáticos em idosos, como os propostos no presente trabalho, ainda não foi realizada. Considerando-se o número crescente da proporção de indivíduos nesta faixa etária e alterações que ocorrem nos vários sistemas associadas ao processo de envelhecimento, em conjunto com os fatores de risco de doenças cardiovasculares, o enfoque atual quanto à realização de exercícios prescrito de forma adequada como meio preventivo de doenças crônico-degenerativas, justifica a realização desta investigação.

Baseando-se em trabalhos anteriores ${ }^{6,13,14}$, hipotetizase que o modelo matemático de Hinkley aplicado aos dados das variáveis estudadas seja eficiente na detecção do LA de um grupo de idosos saudáveis. No entanto, além do modelo de Hinkley, será aplicado o modelo matemático Heteroscedástico e, questiona-se se este também seria eficiente nesta detecção.

Assim, os objetivos deste estudo foram determinar o limiar de anaerobiose (LA) pelo método ventilatório (visual gráfico - padrão ouro), pelos modelos matemáticos de Hinkley e Heteroscedástico, aplicados aos dados de freqüência cardíaca, RMS do sinal mioelétrico e produção de dióxido de carbono, obtidos durante o protocolo de exercício físico dinâmico contínuo do tipo rampa; comparar o LA identificado pelos três métodos e, correlacionar os valores de potência, freqüência cardíaca e consumo de oxigênio no momento do LA identificados pelos modelos matemáticos, com os valores das mesmas variáveis identificados pelo método visual.

\section{MATERIAIS E MÉTODOS}

Na presente investigação foram selecionados 13 homens idosos saudáveis, com padrão de vida ativo, dos quais 9 participaram do estudo, com idade média de 61,4 $\pm 1,78$ anos, massa corporal de $73 \pm 10,8 \mathrm{~kg}$, altura de $1,72 \pm 0,08 \mathrm{~m}$ e IMC de 25,26 $\pm 1,36$. Estes realizavam atividade física regular (corrida, natação, ciclismo e caminhada) em média há 15 anos, 4 dias por semana e com 60 minutos de duração por sessão (em mediana). Previamente à participação, todos voluntários assinaram um termo de consentimento pósinformado e o projeto foi aprovado pelo Comitê de Ética em Pesquisa da Universidade Federal de São Carlos, parecer de número 225/2005.

Os voluntários foram submetidos as seguintes avaliações previamente à aplicação do protocolo experimental: anamnese; exame físico e postural; eletrocardiografia (ECG) convencional de 12 derivações em repouso na posição supina e registro nas derivações MC5, DII e V2 modificadas e aferição de pressão arterial nas posições: repouso supino, sentado e em hiperventilação, exames laboratoriais do perfil lipídico: colesterol total e frações (HDL, LDL, VLDL) e triglicérides; bem como, hemograma completo, glicemia de jejum, ácido úrico, creatinina e uréia. Realizaram também, dois testes de esforço físico em cicloergômetro (Corival Ergometer 400) até a exaustão: a) protocolo I - Inicialmente foi realizado um teste de esforço físico dinâmico em degraus contínuos (TEFDD-c - protocolo I), por um médico cardiologista e 
acompanhado pelo pesquisador, com o propósito de avaliar a capacidade funcional dos voluntários e observar as respostas clínicas e funcionais do sistema cardiovascular, bem como, determinar as variações dos incrementos de potência para o protocolo II. Inicialmente, realizou-se um leve aquecimento, com potência de 4 Watts (W) por um período de 2 minutos e carga inicial de $25 \mathrm{~W}$ por 3 minutos. Posteriormente, foram impostos incrementos de $25 \mathrm{~W}$ a cada 3 minutos, até que o voluntário apresentasse exaustão física ou sinais e sintomas limitantes e/ou atingisse a FC máxima prevista em relação à idade. Anormalidades durante este teste foram também consideradas como critério de exclusão do estudo. b) protocolo II - consistiu num teste de protocolo do tipo em rampa. Este protocolo foi realizado com o objetivo de determinar a capacidade aeróbia funcional e o limiar de anaerobiose ventilatório. Durante este, foram captados e registrados os dados de freqüência cardíaca, variáveis ventilatórias e metabólicas e atividade muscular.

\section{Aquisição das variáveis do estudo}

Os sinais eletrocardiográficos foram captados por um monitor cardíaco de 1 canal (ECAFIX TC500) e processados por meio de um conversor analógico digital (PCI7030/640E, National Instruments), que constitui uma interface entre o monitor cardíaco e o microcomputador (Pentium III 550 $\mathrm{MHz}$ ). Os dados de FC e iR-R, batimento a batimento, foram armazenados no microcomputador. A captação e o processamento dos dados foram feitos por meio de um software específico para a captação de ECG e cálculo dos iR-R em milissegundos ${ }^{15}$.

As variáveis ventilatórias e metabólicas foram obtidas por meio de um sistema computadorizado de análise ergoespirométrica (CPX; Medical Graphics, St Paul, Minnesota, USA) que dispõe de um microcomputador (Pentium III MHz), com uma placa analógico/digital configurada para permitir uma amostragem de 250 valores por segundo por canal (Service Manual MedGraphics); o software utilizado para a captação das variáveis ventilatórias e metabólicas foi o Breeze Suit 5.3.007, que possibilita o armazenamento, processamento e emissão gráfica e relatórios das referidas variáveis em vários formatos.

A eletromiografia de superfície (EMGs) foi obtida a partir de um eletromiógrafo Lynx (Tecnologia Eletrônica Ltda.) e um programa de aquisição de sinais (AqDados 5). Para o procedimento, foram utilizados eletrodos de prata/cloreto de prata, com diâmetro de $1 \mathrm{~cm}$ e distância de 2cm intereletrodos, de centro a centro, acoplados por meio de terminais específicos a um dispositivo diferencial ativo simples adaptado, responsável pela diferenciação entre os dois pólos dos eletrodos. Estes foram fixados no ventre muscular do músculo vasto lateral, mais especificamente no terço distal da medida entre a crista ilíaca ântero-superior e o rebordo lateral patelar do membro a ser avaliado ${ }^{16}$, sendo que, o eletrodo terra foi fixado ao punho do voluntário. Os sinais da EMGs foram processados por um sistema que possui um filtro digital passa banda com freqüências de corte de $20 \mathrm{~Hz}$ para as baixas freqüências e $500 \mathrm{~Hz}$ para as altas freqüências. A freqüência de amostragem do conversor analógico-digital utilizada foi de $2000 \mathrm{~Hz}$, com faixa de tensão de entrada do sinal no canal analógico de 10 a +10 milivolts. Para a análise do RMS (Root Mean Square) do sinal mioelétrico, foram desconsiderados os trechos de repouso do sinal, ou seja, valores de RMS inferiores a 30 microvolts. Este procedimento foi realizado para que o cálculo do RMS fosse feito apenas em relação às contrações musculares.

\section{Métodos de análise para determinação do limiar de anaerobiose}

\section{Método visual gráfico, ventilatório (padrão ouro)}

A análise quantitativa foi realizada inicialmente num gráfico contendo o comportamento das variáveis $\mathrm{VCO}_{2} \mathrm{e} \mathrm{VO}_{2}$, em relação ao intervalo de tempo selecionado para a análise. $\mathrm{O}$ analisador movia o cursor até o ponto em que resposta da $\mathrm{VCO}_{2}$ se elevasse desproporcionalmente ao aumento do $\mathrm{VO}_{2}$ (perda do paralelismo entre estas variáveis) ${ }^{5}$. Este método de determinação do LAV foi considerado o padrão ouro do presente estudo, e foi utilizado na comparação com as demais metodologias.

Para a determinação do limiar de anaerobiose ventilatório (LAV), foi selecionado o intervalo dos dados do teste em rampa onde se observava o início da resposta das variáveis ventilatórias ao incremento de potência até o momento do ponto de compensação respiratória (aumento desproporcional da ventilação em relação à produção de gás carbônico - PCR) ou então, até o final do exercício (quando não se atingia o PCR). Foi calculada a média dos valores obtidos de três determinações do LA independentes.

\section{Método do modelo matemático de Hinkley $(H)$ e modelo matemático Heteroscedástico $(R)$}

Estes modelos foram aplicados às variáveis freqüência cardíaca, RMS do sinal mioelétrico e produção de gás carbônico. São modelos de regressão linear que determinam o ponto de mudança de uma série de dados pelo método da máxima verossimilhança. Para se obter um ajuste adequado, é necessário que as respostas das variáveis escolhidas apresentem apenas uma mudança de inclinação entre as retas, resultante dos respectivos comportamentos. Desta maneira, o mesmo intervalo selecionado para o padrão ouro foi adotado para os valores das variáveis $\mathrm{FC}, \mathrm{RMS}$ e $\mathrm{VCO}_{2}$.

Para análise estatística de significância dos dados foi utilizado o teste não-paramétrico de Friedman para medidas repetidas seguido de técnicas de comparações múltiplas com teste post-hoc de Dunn, que informa em que nível se deu a significância entre os dados. Foi aplicado ainda o teste de correlação de Spearman. O nível de significância estabelecido para todos os testes foi de $5 \%$. 


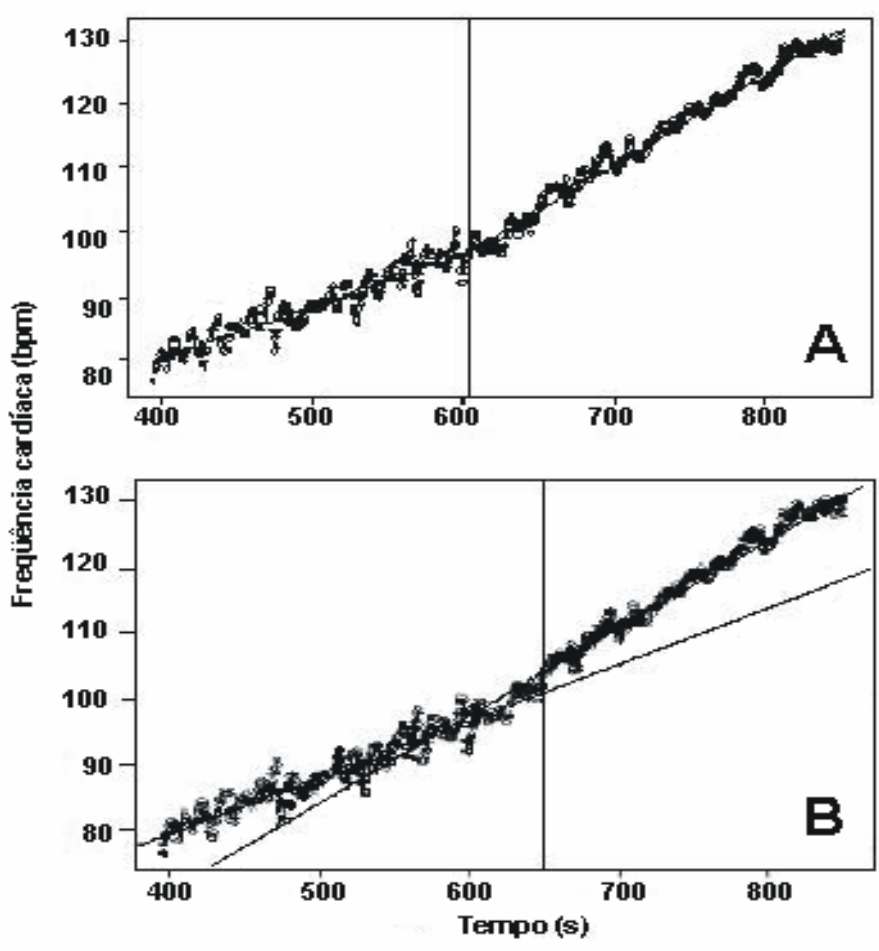

Figura 1. Ilustração da análise dos modelos matemáticos (Hinkley em A e Heteroscedástico em B) aplicados aos dados de freqüência cardíaca, batimento a batimento, de um dos voluntários estudados. A reta vertical determina o ponto de mudança do comportamento da freqüência cardíaca. O tempo na parte inferior do gráfico, determinado por esta reta, foi considerado o tempo de ocorrência do limiar de anaerobiose.

\section{RESULTADOS}

Os valores medianos de potência, $\mathrm{FC}$ e $\mathrm{VO}_{2}$ no pico do esforço foram $149 \mathrm{~W}, 144 \mathrm{bpm}$ e 25,58ml/kg/min, respectivamente. Na tabela 1 estão apresentados os valores de potência, $\mathrm{FC} \mathrm{e} \mathrm{VO}_{2}$, identificados pelos modelos visual gráfico e matemáticos de Hinkley e Heteroscedástico.

Na avaliação da adequação dos modelos matemáticos aplicados aos dados de FC para a determinação do LA, o presente estudo mostra que os modelos $\mathrm{R}$ e $\mathrm{H}$ apresentam correlação em relação ao padrão ouro quando comparados os valores de FC ( $r=0,81, p=0,006 ; r=0,79, p=0,01)$ e $\mathrm{VO}_{2}(\mathrm{r}=0,68$ e $0,68, \mathrm{p}=0.042$, para ambos), respectivamente. Quando aplicados aos valores de $\mathrm{VCO}_{2}$, houve significância quando comparados os valores de FC em relação ao padrão ouro $(r=0,92, p=0002$, para o modelo $\mathrm{R} ; \mathrm{r}=076, \mathrm{p}=0,015$, para o modelo H). Já quando aplicados aos dados de RMS, somente o modelo $\mathrm{H}$ apresentou correlação em relação ao padrão ouro, quando comparados os valores de potência $(r=0,68, p=0,041)$.

\section{DISCUSSÃO}

No presente estudo, os modelos matemáticos foram aplicados aos valores de FC, RMS e $\mathrm{VCO}_{2}$, obtidos durante o teste de esforço físico dinâmico contínuo do tipo rampa, com o objetivo de identificar o ponto de mudança no padrão de comportamento destas séries de dados.

Conconi et al. ${ }^{17}$, apresentaram um trabalho propondo avaliar a perda da linearidade da FC em função da potência durante protocolo de exercício incremental, como indicador não-invasivo do LA, comparativamente à coleta de lactato sangüíneo. Estes autores utilizaram o método visual de determinação do LA e mesmo encontrando relação entre o ponto de deflexão da FC, em função da potência, com o LA, este estudo tem sido questionado, frente a dificuldades encontradas na reprodutibilidade deste modelo em alguns voluntários ${ }^{18}$. Por outro lado, autores, também usando o método visual, mas comparativamente ao método ventilatório, observaram que a quebra da linearidade da FC coincide com o ponto de compensação respiratória ${ }^{8}$. Ainda, nos resultados de Vachon, Basset e Clarke ${ }^{19}$, utilizando uma equação polinomial de terceira ordem para identificar o ponto de quebra da FC em homens treinados (30,8 \pm 5,9 anos), durante teste de esforço físico incremental, em esteira, encontrou um comportamento linear desta variável durante todas as velocidades impostas. Também em esteira ergométrica, Lucía et al. ${ }^{20}$, aplicando um algoritmo de regressão linear, encontraram quebra da linearidade da FC em apenas 31\% dos idosos treinados estudados (62 \pm 1 anos). Com o mesmo método,

Tabela 1. Valores de potência, $\mathrm{FC} \mathrm{e} \mathrm{VO}_{2}$ no limiar de anaerobiose identificado pelos modelos visual gráfico e matemáticos de Hinkley e Heteroscedástico, aplicados às variáveis FC, RMS e $\mathrm{VCO}_{2}$. Nível de significância de p<0,05.

\begin{tabular}{lccccccc}
\hline & \multicolumn{3}{c}{ HETEROSCEDÁSTICO } & \multicolumn{3}{c}{ HINKLEY } \\
& Visual & FC & RMS & VCO $_{2}$ & FC & RMS & VCO $_{2}$ \\
\hline Potência & 71 & 82 & $85^{*}$ & 80 & 77 & 78 & $67^{*}$ \\
FC & 99 & 105 & 105 & 105 & 101 & 104 & 99 \\
VO $_{2}$ & 12,6 & 13,7 & $14,4 *$ & 12,25 & 12,5 & 12,6 & $10,35^{*}$ \\
\hline
\end{tabular}


Lucía et al. ${ }^{21}$, avaliou o comportamento da FC de homens ciclistas profissionais (26 \pm 1 anos) , durante protocolo de esforço físico dinâmico em degraus contínuos (25W/min), mostrou que o ponto de deflexão da FC em função da potência ocorreu em torno de $88 \%$ da máxima, em 56\% dos voluntários.

Os resultados encontrados no presente estudo diferem dos citados acima, podendo estas diferenças estar relacionadas ao protocolo de exercício utilizado, ao grupo estudado e à metodologia de análise empregada.

No estudo de Hoffman et al. ${ }^{9}$ e Bunc et al. ${ }^{10}$, foi aplicado um modelo matemático de ajuste linear aos dados de FC coletados durante o teste de exercício físico dinâmico incremental, que detectava o ponto onde a resposta da FC perdia a linearidade em relação ao aumento de potência. Os autores referem que este ponto de quebra foi fortemente correlacionado com o LA determinado pela metodologia de concentração de lactato sanguíneo. Apesar de no presente estudo a metodologia de referência ter sido a análise visual gráfica do comportamento das variáveis ventilatórias e metabólicas e com amostra diferente, os resultados estão de acordo com os observados por esses dois estudos, em relação à utilização de algoritmos matemáticos para a identificação do LA, quando comparados com metodologias tradicionais.

Durante o exercício incremental proposto, o comportamento da $\mathrm{VCO}_{2}$, foi similar ao da FC. A VCO aumentou linearmente com incremento de potência, $\mathrm{FC}$ e $\mathrm{VO}_{2}$ e, numa certa intensidade, ocorre um aumento desproporcional dos valores encontrados, concomitante a um aumento adicional da VE, que pode ser explicado pela liberação adicional de $\mathrm{CO}_{2}$ (cerca de 2,5 vezes) resultante da dissociação do ácido carbônico, formado a partir do tamponamento do lactato pelo bicarbonato sanguíneo ${ }^{1}$.

Dessa forma, os resultados encontrados mostram que a mudança no padrão do comportamento da $\mathrm{FC}$ e $\mathrm{VCO}_{2}$, durante exercício físico dinâmico progressivo, pode ocorrer em instantes próximos às mudanças do comportamento ventilatório e metabólico, sugerindo que a metodologia pode ser utilizada na determinação do LA, uma vez que os ajustes providos pelo sistema cardiorrespiratório são mediados conjuntamente.

No presente estudo, a resposta da atividade do músculo vasto lateral, avaliada a partir do índice RMS em função do tempo, durante exercício físico dinâmico incremental em cicloergômetro, mostra um aumento linear da amplitude da EMGs até uma certa potência, a partir da qual observou-se uma perda desse comportamento. Esses dados são concordantes com outros estudos ${ }^{22,23}$. A mudança do padrão de resposta da EMGs deve-se provavelmente a uma associação entre o aumento no recrutamento e na freqüência de disparo das unidades motoras das fibras musculares de contração rápida. Associação esta que pode estar relacionada ao esgotamento progressivo das fibras musculares oxidativas, de contração lenta, em intensidades elevadas de exercício, sendo necessário um recrutamento adicional das fibras glicolíticas, de contração rápida, para a manutenção do trabalho exigido ${ }^{25}$. Fibras glicolíticas são menos eficientes e menos econômicas, contribuindo para o acúmulo de lactato sanguíneo ${ }^{12}$.

Nos resultados encontrados por Lucía et al. ${ }^{20}$, avaliando ciclistas profissionais durante exercício físico em carga constante correspondente a $80 \%$ do $\mathrm{VO}_{2 \text { máx }}$ o crescente aumento dos valores das variáveis $\mathrm{FC}, \mathrm{VCO}_{2}, \mathrm{VE}, \mathrm{VE} / \mathrm{VCO}_{2}$ e $\mathrm{VE} / \mathrm{VO}_{2}$, não foi acompanhado do aumento do RMS do sinal mioelétrico, sugerindo que, em atletas treinados, o aumento dos valores das variáveis ventilatórias e metabólicas, associados ao LA, não parece estar relacionado à concomitante mudança no padrão de recrutamento das fibras musculares.

Hug et al. ${ }^{24}$ avaliaram a resposta de oito grandes músculos de perna e coxa de ciclistas profissionais durante teste de esforço incremental (26W/min a partir de $100 \mathrm{~W})$. Os pontos de quebra do comportamento do RMS do sinal mioelétrico ocorreram em $100 \%$ dos voluntários para o músculo vasto lateral e em 50\% para a porção lateral do gastrocnêmio, enquanto a avaliação dos músculos vasto lateral e bíceps da coxa permitem a determinação de dois pontos de quebra no comportamento do RMS, um abaixo do LA e outro coincidente com o ponto de compensação respiratória (PCR). Lucía et al. ${ }^{11}$, aplicando um protocolo incremental, também encontraram dois pontos de quebra no RMS e que ocorreram em 52\% (abaixo do LA) e 86\% (similar ao PCR) da potência máxima, respectivamente.

Tal fato pôde ser explicado pelo fato da ocorrência do LA ser resultado do início do desequilíbrio entre a produção e utilização de lactato por todos os músculos envolvidos na atividade física, os quais produzem lactato em diferentes tempos. O limiar da EMG de um único músculo pode ser detectado, mas ao se utilizar o método de análise de gases expirados isto não é possível, tendo em vista que estes parâmetros obtidos refletem as respostas metabólicas de todos os músculos envolvidos.

Embora não havendo diferença significante entre os valores de LA identificados, as correlações entre os valores de potência, FC e $\mathrm{VO}_{2}$ no LA obtidas pela aplicação dos modelos matemáticos aos dados de RMS foram inferiores àquelas onde foram utilizados os dados $\mathrm{FC} \mathrm{e} \mathrm{VCO}_{2}$. Sakabe e Marães ${ }^{13}$, avaliando homens saudáveis meia-idade sedentários e jovens ativos, respectivamente, os valores de LA identificados pelo modelo de Hinkley não foram estatisticamente diferentes do valor obtido pelo padrão ouro e foram encontradas boas correlações entre as variáveis potências, FC e $\mathrm{VO}_{2}$ no LA, identificado pelas variáveis FC, RMS e $\mathrm{VCO}_{2}$. No entanto os menores valores de correlação foram encontrados na aplicação do modelo aos dados de RMS. Os nossos dados são concordantes aos de Sakabe ${ }^{6}$ e Marães ${ }^{13}$, no que se refere aos menores valores de correlação na aplicação dos modelos matemáticos H e R aos dados de RMS, em relação aos identificados pelo padrão ouro. 


\section{CONCLUSÕES}

Dessa forma, no presente estudo, por meio de ambos os modelos matemáticos foi possível identificar valores de LA semelhantes aos identificados pelo método padrão ouro, sendo que os modelos ajustaram-se melhor e igualmente, aos dados de FC. Ressalta-se a importância do presente trabalho por avaliar a aplicação de modelos matemáticos na identificação do limiar de anaerobiose de idosos, o que possibilitará uma prescrição de atividade física segura e adequada, a partir de uma ferramenta de baixo custo e de fácil obtenção, a freqüência cardíaca.

Apoio: Capes, CNPq, FAPESP.

\section{REFERÊNCIAS BIBLIOGRÁFICAS}

1. Wasserman K, Hansen JE, Sue D, Whipp BJ, Casaburi R. Principles of exercise testing \& interpretation: including pathophysiology and clinical applications. 3 ed. Philadelphia: Lippincott Williams \& Wilkins, 1999.

2. Chacon-Mikahil MPT, Forti VAM, Catai AM, Szrajer JS, Golfetti R, Martins LEB, et al. Cardiorespiratory adaptations induced by aerobic training in middle-aged men: the importance of a decrease in sympathetic stimulation for the contribution of dynamic exercise tachycardia. Braz J Med Biol Res 1998; 31(5): 705-712.

3. Catai AM, Chacon-Mikahil MPT, Martinelli FS, Forti VAM, Silva E, Golfetti R, et al. Effects of aerobic exercise training on heart rate variability during wakefulness and sleep and cardiorespiratory responses of young and middle-aged healthy men. Braz J Med Biol Res 2002; 35 (6): 741-752.

4. Beaver WL, Wassernan K, Whipp BJ. A new method for detecting anaerobic threshold by gas exchange. J Appl Physiol 1986; 60(6): 2020-2027.

5. Crescêncio JC. Determinação do limiar de anaerobiose ventilatório no exercício físico dinâmico em indivíduos sadios. Comparação entre métodos obtidos por análise visual e modelos matemáticos [dissertação]. Ribeirão Preto (SP): Universidade de São Paulo.; 2002.

6. Sakabe DI. Quantificação do limiar de anaerobiose durante exercício físico dinâmico: análise da resposta de variáveis cardiorrespiratórias e musculares [dissertação]. São Carlos (SP): Universidade Federal de São Carlos.; 2004.

7. Marães VRFS, Silva E, Catai AM, Novais LD, Moura MAS, Oliveira L, et al. Identification of anaerobic threshold using heart rate response during dynamic exercise. Braz J Med Biol Res 2005; 38: 731-735.

8. Ribeiro JP, Fielding A, Hughes V, Black H, Bochese MA, Knuttegen HG. Heart rate break point may coincide with the anaerobic threshold and not the aerobic threshold. Int J Sports Med 1985; 6 (4): 220-224.

9. Hoffman P, Bunc V, Leitner H, Pokan R, Gaisl G. Heart rate threshold related to lactate turn point and steady-state exercise on a cycle ergometer. Eur J Appl Physiol 1994; 69: 132-139.

10. Bunc V, Hoffman P, Leitner H, Gaisl G. Verification of heart rate threshold. Eur J Appl Physiol 1995; 70: 263-369.
11. Lucía A, Sánches O, Carvajal A, Chicharro JL. Analysis of the aerobic-anaerobic transition in the elite cyclists during incremental exercise with the use of electromyography. Br J Sports Med 1999; 33: 178-185.

12. Bearden SE, Mofatt RJ. Leg electromyography and the VO2power relationship during bicycle ergometry. Med Sci Sports Exer 2001; 33 (7): 1241-1245.

13. Marães VRFS. Determinação do limiar de anaerobiose a partir da análise da freqüência cardíaca, da eletromiografia de superfície e das variáveis ventilatórias e metabólicas durante exercício físico dinâmico [tese]. São Carlos (SP): Universidade Federal de São Carlos.; 2004.

14. Pessoti ER. Determinação do limiar de anaerobiose a partir da resposta da frequiência cardíaca, da atividade mioelétrica, do consumo de oxigênio ao exercício físico dinâmico de homens de meia idade saudáveis e de hipertensos [dissertação]. Piracicaba (SP): Universidade Metodista de Piracicaba.; 2005.

15. Silva E. Catai AM, Trevelin LC, Guimarães JO, Silva Jr. LP, Silva LMP, et al. Design of a computerized system to evaluate the cardiac function during dynamic exercise. Anals of World Congress of Medical Physics and Biomedical Engeneering; 1994. p. 409.

16. Freriks B, Hermens HJ. SENIAM 9. European Recommendations for Surface Electromyography, ISBN: 90-75452-14-4 (CDROM). Roessing Research and Development, 1999.

17. Conconi F, Ferrari M, Ziglio PG, Droghetti P, Codeca L. Determination of the anaerobic threshold by a noninvasive fieldtest in runners. J Appl Physiol 1982; 52: 869-873.

18. Oscelik O, Kelestimur H. Effects of acute hypoxia on the determination of anaerobic threshold using the heart rate-work rate relationships during incremental exercise tests. Physiol Res 2004; 53: 45-51.

19. Vachon JA, Basset DR Jr, Clarke S. Validity of the heart rate deflection point as a predictor of lactate threshold during running. J Appl Physiol 1999; 87(1): 452-459.

20. Lucía A, Carvajal A, Pérez M, Boraita A, Serratosa L, Chicharro $\mathrm{JL}$. Heart rate response during incremental exercise in master runners. Jap J Physiol 2000; 50: 155-158.

21. Lucía A, Hoyos J, Santalla A, Pérez M, Carvajal A, Chicharro JL. Lactic acidosis, potassium, and the heart rate deflection point in professional road cyclists. Br J Sports Med 2002; 36: 113-117.

22. Nagata A, Muro M, Moritani T, Yoshida T. Anaerobic threshold determination by blood lactate and signals myoeletric. Jap J Physiol 1981; 31: 585-597.

23. Jammes Y, Zattara-Hartmann MC, Caquelard F, Arnaud S, Tomei C. Electromyographic changes in vastus lateralis during dynamic exercise. Muscle Nerve 1997; 20: 247-49.

24. Hug F, Faucher M, Kipson N, Jammes Y. EMG signs of neuromuscular fatigue related to the ventilatory threshold during cycling exercise. Clin Physiol Funct Imaging 2003; 23: 208-214.

25. Petrofsky JS. Frequency and amplitude analysis of the EMG during exercise on the bicycle ergometer. Eur J Appl Physiol 1979; 41: 1-15. 